IdeAs

Idées d'Amériques

$2 \mid 2012$

Universités d'Amériques et construction des savoirs

\title{
Sociohistoire des Black Studies Departments
}

Historia Social de los Departamentos de Estudios Negros

Black Studies Departments: A Sociohistory

\section{Caroline Rolland-Diamond}

\section{OpenEdition}

\section{Journals}

Édition électronique

URL : https://journals.openedition.org/ideas/266

DOI : 10.4000/ideas.266

ISSN : 1950-5701

Éditeur

Institut des Amériques

Référence électronique

Caroline Rolland-Diamond, "Sociohistoire des Black Studies Departments », IdeAs [En ligne], 2 | 2012, mis en ligne le 22 juin 2012, consulté le 19 octobre 2022. URL : http://journals.openedition.org/ideas/ 266 ; DOI : https://doi.org/10.4000/ideas.266

Ce document a été généré automatiquement le 19 octobre 2022

\section{(ब) $(\oplus \Theta$}

Creative Commons - Attribution - Pas d'Utilisation Commerciale - Pas de Modification 4.0 International - CC BY-NC-ND 4.0

https://creativecommons.org/licenses/by-nc-nd/4.0/ 


\title{
Sociohistoire des Black Studies Departments
}

\author{
Historia Social de los Departamentos de Estudios Negros \\ Black Studies Departments: A Sociohistory
}

Caroline Rolland-Diamond

1 En mars 1969, la première Faculté d'études ethniques (School of Ethnic Studies, aujourd'hui College of Ethnic Studies) des États-Unis voyait le jour à San Francisco State College, à l'issue d'une grève qui avait paralysée l'établissement public californien pendant cinq mois. Cette création venait compléter celle du Département d'études noires (Black Studies Department) créé à l'automne 1968 et qui allait être devenir partie intégrante de cette nouvelle Faculté. La longueur de la grève menée par le Third World Liberation Front (Front de libération du Tiers monde) et la personnalité intransigeante du président du College, S.I. Hayakawa, ont contribué à faire connaître les événements de San Francisco dans le monde entier ${ }^{1}$. Au-delà du double caractère pionnier et exceptionnel du cas de San Francisco State College, les événements qui ont agité ce campus californien sont représentatifs d'un mouvement plus vaste de transformation de l'institution universitaire par la création de nouveaux départements d'études noires dans 200 colleges et universités environ dans le pays entre 1968 et $1971^{2}$.

2 Tantôt appelées «Black Studies Departments ", "Africana Studies Departments », ou encore "Afro-American Studies Departments", ces nouvelles unités académiques avaient pour point commun d'introduire l'étude systématique de l'histoire, de la culture et des expériences de la communauté noire américaine dans l'université. À cette diversité de désignations, s'ajoute aussi une diversité de structures : alors que ces créations prirent souvent la forme de départements indépendants, les unités furent parfois intégrées à un département existant, tandis que leurs enseignants bénéficiaient fréquemment d'une double affectation dans deux structures différentes, par exemple le département d'histoire et celui d'études noires. Quelles que furent les spécificités de chaque structure, toutes étaient directement inspirées de l'idéologie du black power et visaient non seulement à modifier et à enrichir le contenu des enseignements dispensés par les universités en y intégrant la contribution des Afro-Américains mais aussi, et 
plus fondamentalement, à transformer le fonctionnement de l'institution universitaire en accroissant le nombre d'étudiants, d'enseignants et d'administrateurs noirs (pour rendre l'université plus représentative de la société américaine dans son ensemble) et en y favorisant l'émergence de nouveaux savoirs en lien direct avec la communauté noire extérieure.

3 Au début des années 1970, lorsque le mouvement du black power connut un reflux généralisé sous le double effet des limites de son action dans la communauté noire et de la répression politique qui s'abattit sur lui, les départements d'études noires devinrent l'un de ses derniers bastions, ou plus précisément l'un des derniers vestiges de son influence. Se posa alors la question de leur pérennité au sein d'une institution globalement restée inchangée après une décennie de protestation lancée au moment du Free Speech Movement de Berkeley, autrement dit la question de l'adaptation du discours idéologique radical qui leur a donné naissance aux exigences propres aux disciplines académiques et à l'évolution de l'institution universitaire dans un paysage politique et social marqué par la montée du conservatisme.

Répondre à cette double question suppose de se pencher plus précisément sur les conditions historiques de l'apparition de l'idéologie du black power sur les campus et de la fondation des Black Studies Departments dans les dernières années du moment 68 avant de réfléchir aux défis spécifiques posés par l'institutionnalisation de ces départements dans le contexte du retour en force du conservatisme politique à partir des années Nixon.

\section{De l'émergence du black power sur les campus}

Transcription ouvertement revendiquée de l'idéologie du Black Power sur les campus, les départements d'études noires et/ou ethniques qui virent le jour à l'extrême fin des années soixante sont le produit d'un mouvement social et politique radical qui se transforma en s'adaptant aux réalités de l'une des institutions qu'il essaya de changer : l'université. Ce faisant, il laissa derrière lui ce qui constitue sans doute son héritage le plus durable, comme l'a bien montré le sociologue Fabio Rojas, dans son ouvrage majeur From Black Power to Black Studies.

6 Concept malléable né d'un sentiment de désillusion et de frustration devant l'absence de transformation des conditions de vie des habitants des ghettos des grandes villes du Nord après plus de dix ans de lutte pour les droits civiques, l'idéologie du black power se déclinait sous plusieurs variantes - séparatisme, nationalisme noir ou pluralisme culturel - constituant toutes, au-delà de leurs différences, une rupture par rapport à l'approche défendue par le mouvement pour les droits civiques. À la tactique de nonviolence inconditionnelle prônée par Martin Luther King, les tenants du black power préféraient l'idée, défendue de manière très éloquente par Malcolm $\mathrm{X}^{3}$, du droit à l'auto-défense. En outre, alors que l'objectif ultime du mouvement pour les droits civiques était l'intégration individuelle dans la société américaine, basée sur l'égalité des droits de chacun et l'idéal d'une société colorblind, les partisans du nationalisme noir cherchaient, quant à eux, à obtenir l'égalité en tant que groupe, dans le respect de leur différence et en rejetant toute assimilation dans le melting pot du conformisme anglo-saxon. En lieu et place de la vision d'une société d'individus sans race, ou au-delà des races, développée par le mouvement pour les droits civiques, les nationalistes noirs voyaient dans les États-Unis une société composée de groupes ethno-raciaux possédant 
différents degrés de pouvoir, de privilèges et d'avantages. Cette reconnaissance du pluralisme ethno-racial et culturel remplaçait l'effacement de la différence défendue par les militants des droits civiques par la conscience de la différence ${ }^{4}$ et par la revendication de la transformation des institutions du pays en vue du renforcement $\mathrm{du}$ pouvoir de chaque groupe minoritaire. Particulièrement chères aux étudiants noirs, ces deux dernières idées les incitèrent à se regrouper dans des black student unions qui se mirent, à partir de l'année 1968, à réclamer, entre autres, la création de départements d'études noires dans leurs universités. En introduisant, par leurs revendications, l'idéologie du black power sur les campus, les étudiants nationalistes noirs bousculèrent l'institution universitaire en la forçant à s'adapter mais, ce faisant, ils furent eux mêmes contraints d'adapter leur discours et leurs exigences aux défis immédiats et à plus long terme que posait désormais l'institutionnalisation de ce mouvement social radical.

7 Analysant ce processus de transformation, Rojas montre que la création des départements d'études noires a été rendue possible par la conjonction de trois facteurs clés : tout d'abord, la désillusion que ressentirent de nombreux jeunes noirs face au mouvement pour les droits civiques les convainquit que les institutions du pays ne changeraient que si des mesures radicales étaient prises; dans le contexte de ce désenchantement, de nouvelles organisations, tel le Black Panther Party, virent le jour pour promouvoir l'idéologie du Black Power. Très populaires auprès des jeunes, ces groupes devinrent une ressource cruciale pour les étudiants noirs qui, troisième facteur, venaient d'être admis pour la première fois en nombre conséquent dans des universités essentiellement blanches. Pour ces jeunes, la réforme du contenu des enseignements dispensés ne suffisait pas à changer en profondeur l'institution universitaire; seule la création de nouveaux départements spécifiquement consacrés aux études afro-américaines pouvait permettre d'atteindre cet objectif ${ }^{5}$.

\section{Précédents historiques}

8 L'idée d'introduire la contribution des noirs américains dans l'enseignement de l'histoire des États-Unis n'était pas chose nouvelle ${ }^{6}$. Les premières tentatives avaient vu le jour dès l'époque de la Reconstruction qui suivit la guerre de Sécession avec, d'une part, la création d'écoles noires pour alphabétiser les enfants et les adultes récemment affranchis et leur apprendre à être fiers de leurs origines et, d'autre part, l'établissement d'universités noires, aujourd'hui appelées les Historically Black Colleges and Universities (ou HCBU), telles Howard University, Fisk University ou Morehouse College fondées juste après la guerre de Sécession pour offrir un accès à un enseignement de type liberal college adaptés à l'expérience spécifique des jeunes afroaméricains. De même, les critiques d'un système d'enseignement supérieur dominé par les blancs qui se contente de reproduire la hiérarchie raciale de la société environnante avaient déjà été exprimées haut et fort au début du vingtième siècle notamment par W.E.B. DuBois ou dans les travaux de Carter G. Woodson, éminent historien et fondateur du Journal of Negro History. Dans son ouvrage le plus connu, The Mis-Education of the Negro, ce dernier expliquait que les universités étaient trop occupées à éduquer les étudiants blancs et négligeaient les besoins des noirs. Accusant les étudiants afroaméricains de passer trop de temps à étudier l'histoire d'autres races parce qu'ils partageaient le préjugé commun à cette époque que leur propre histoire n'était pas aussi importante, il expliquait que c'était précisément cette croyance qui maintenait les 
noirs dans une position subalterne ${ }^{7}$. Exprimées à une période où la prise de conscience des problèmes posés par la persistance de la ségrégation dans la société américaine n'en était qu'à ses premiers balbutiements, ces critiques à la résonance moderne ne permirent pas de transformation immédiate de l'institution universitaire, d'autant que le nombre extrêmement limité d'étudiants noirs sur les campus américains ne leur permettait nullement de faire entendre leurs griefs et leurs légitimes aspirations. Mais ces critiques servirent de précédents historiques essentiels et de références intellectuelles aux générations suivantes d'activistes qui se mobilisèrent à partir de la seconde guerre mondiale pour revendiquer la justice raciale et sociale pour les afroaméricains dans toutes les sphères de la société et, à la fin des années soixante, à l'université.

\section{Le nouvel étudiant noir : entre campus et ghetto}

Le changement social décisif qui permit l'émergence des départements d'études noires dans les universités américaines fut la déségrégation du système éducatif que le mouvement pour les droits civiques entraîna. Pour la toute première fois, le nombre d'étudiants noirs sur des campus très majoritairement blancs allait suffisamment augmenter pour permettre l'expression de revendications identitaires. Les HCBU devinrent, quant à eux, des centres essentiels de la lutte pour la déségrégation en fournissant les troupes de choc du mouvement des sit-ins lancé en 1960 et en inspirant toute une génération d'étudiants à créer des clubs et des associations dédiées à la réforme de leur campus. Une nouvelle génération d'activistes, ayant découvert la politique dans les luttes du mouvement pour les droits civiques dont elle avait connu les espoirs, les succès mais aussi les déceptions, fit son entrée sur les campus du pays à partir de la deuxième moitié des années soixante, dotée d'une nouvelle attitude militante et de la volonté de transformer l'institution universitaire en faisant entendre leur voix ${ }^{8}$.

Jusqu'à la mise en place des politiques d'affirmative action dans les années 1970, le nombre d'étudiants noirs sur les campus resta très limité, comme l'illustre bien la situation dans une ville comme Chicago ${ }^{9}$. Alors que la deuxième métropole du pays comprenait une forte population afro-américaine - près de 23 pour cent du total en 1960 et plus de 32 pour cent en $1969^{10}$ - l'Université de l'Illinois à Chicago ne comptait que 80 étudiants undergraduate noirs sur 2000 en 1969, soit 4 pour cent seulement du total. Une situation similaire se retrouvait sur le campus de l'Université de Chicago où la proportion d'étudiants noirs s'élevait aussi à $4 \%$ en septembre $1967^{11}$. Mais en chiffres absolus, le contingent noir s'élevait désormais à plusieurs dizaines voire centaines de personnes, un nombre suffisant pour pouvoir monter des actions de protestation avec un espoir de réussite. Comme l'ont bien montré les théoriciens des mouvements sociaux, Doug McAdam en tête, l'augmentation du nombre d'étudiants afro-américains créa les conditions nécessaires à la possibilité d'émergence d'un activisme parmi ce groupe d'étudiants. En effet, tant qu'ils ne représentaient qu'une simple poignée d'étudiants à l'échelle d'un campus de plusieurs centaines ou milliers d'étudiants, il leur était impossible de donner voix à leurs griefs ${ }^{12}$. Une fois les ressources nécessaires à l'émergence de l'activisme réunies, il ne manquait plus qu'un facteur déclenchant pour que cette dernière se produise. Les émeutes urbaines des quartiers défavorisés et la popularité croissante de l'idéologie du Black Power jouèrent ce rôle de catalyseur. 
11 Membres de la même génération que les étudiants blancs qui protestaient contre la guerre du Vietnam et la complicité des universités avec le complexe militaro-industriel depuis le milieu des années soixante, les nouveaux étudiants afro-américains partageaient avec ceux-ci la même volonté de remise en cause des valeurs traditionnelles et de l'autorité, ainsi que la quête d'une identité individuelle et collective en accord avec leurs nouvelles valeurs. Mais leur situation était particulière en ce sens qu'ils étaient aussi directement inspirés par la radicalisation de la lutte des noirs dans les quartiers défavorisés des grandes villes du Nord depuis le milieu des années soixante, sous l'influence grandissante de l'idéologie du Black Power. À cette période, la grande majorité des étudiants afro-américains était issue de milieux défavorisés, y compris ceux inscrits dans les universités prestigieuses telles que l'Université de Chicago. La loi fédérale sur l'enseignement supérieur (Higher Education Act $)^{13}$ de 1965 , destinée à faciliter l'accès à l'enseignement supérieur des étudiants issus de milieux défavorisés, et les efforts d'un nombre croissant d'universités pour recruter des étudiants noirs issus de ces milieux provoquèrent en effet une transformation de l'étudiant afro-américain "type " à la fin des années 1960. Avant cette période, les étudiants afro-américains avaient beaucoup plus tendance à venir de milieux de classe moyenne et, de ce fait, avaient moins de chance d'être marqués par les conditions de vie dans les ghettos. Ce changement démographique conduisit à l'apparition d'une nouvelle «conscience noire » sur les campus américains de la fin des années 1960 qui participa de l'émergence d'un mouvement de protestation intéressé par les questions d'inégalité et de discrimination raciales ${ }^{14}$. Issus des mêmes milieux, les étudiants afroaméricains ressentaient donc les mêmes frustrations, angoisses et colères que leurs pairs dans leurs quartiers, à la seule différence que leur statut d'étudiants leur conférait une position privilégiée sur l'échelle de la mobilité sociale par rapport à ces derniers.

12 Si les étudiants noirs étaient sur la voie d'obtenir un diplôme, sanctionnant une formation supérieure gage, dans l'Amérique des années soixante, d'une insertion réussie dans la vie professionnelle, ils n'en restaient pas moins intéressés par le devenir des quartiers dont ils étaient originaires et de la communauté noire en général. Les études menées sur ce sujet ${ }^{15}$ montrent que, contrairement à l'étudiant blanc contestataire du moment 68, l'étudiant afro-américain ne cherchait pas à détruire l'institution universitaire, mais plutôt à la réformer pour qu'elle réponde mieux à ses besoins : acquérir les compétences nécessaires à l'amélioration de la situation de sa communauté. Une telle réforme passait à ses yeux par la reconnaissance du racisme institutionnel de l'université, l'augmentation du nombre d'étudiants noirs, le recrutement d'enseignants et d'administrateurs noirs, la création et le contrôle de départements d'études noires, l'ouverture des installations universitaires aux habitants des quartiers voisins, ou encore le droit d'organiser certaines manifestations culturelles spécialement destinées aux étudiants afro-américains. La revendication de création des départements d'études noires doit donc se comprendre comme un élément essentiel, mais un élément parmi d'autres de la transformation de l'institution universitaire, sous l'influence de l'idéologie nationaliste noire, pour la mettre au service de la communauté noire, sur le campus et, plus fondamentalement, au-delà, dans les ghettos. Cet ancrage communautaire fort est essentiel pour comprendre la forme que prirent les départements d'études noires créés, sous la pression, entre 1968 et 1971, et les défis que ces nouvelles unités disciplinaires rencontrèrent dans les premières années de leur existence. 


\section{La création de départements d'études noires : des revendications aux premiers défis}

13 Première étape vers une éradication du racisme des établissements d'enseignement supérieur, l'augmentation du nombre d'étudiants afro-américains admis à l'université revendiquée par les jeunes nationalistes noirs sur les campus ne pouvait suffire à transformer radicalement l'institution universitaire. D'où les autres revendications des étudiants noirs que l'on retrouve sur les nombreux campus touchés par ce mouvement de revendication, de San Francisco State College à Brown University (à Providence) en passant par Harvard, Temple University, Cornell University, l'Université de l'Illinois ou encore Howard University à Washington D.C. ${ }^{16}$ : la création de programmes d'études noires et le recrutement d'enseignants et d'administrateurs issus, eux aussi, de la communauté afro-américaine.

\section{Les nouveaux départements d'études noires : premier pas vers la « libération » de la communauté}

Les nouveaux cours, dispensés par des enseignants noirs, devaient devenir partie intégrante du programme de tronc commun des différentes institutions et les notes obtenues compter en vue de l'obtention du diplôme final. La liste des revendications étudiantes se poursuivait ensuite par l'adoption d'une politique concertée de recrutement d'enseignants noirs dans l'ensemble des départements de l'université. Partant du principe que l'expérience afro-américaine ne pouvait être correctement enseignée que par ceux qui l'avaient vécue, les tenants de la création de département d'études noires réclamaient le recrutement d'enseignants afro-américains, de préférence ayant grandi dans des quartiers pauvres et défenseurs actifs de la cause de la communauté noire américaine. L'objectif était de faire découvrir, aux étudiants noirs et blancs, et par leur intermédiaire, à l'ensemble de la société américaine, la spécificité et la richesse de la contribution des afro-américains à la société américaine et de leur donner ainsi un nouveau cadre référentiel, à l'aune duquel il devenait enfin possible de juger les deux communautés - noire et blanche - sur un pied d'égalité. Mettant fin à la prétention du modèle occidental blanc à être l'étalon universel de l'expérience humaine, les cours et/ou départements d'études noires étaient envisagés comme le premier pas de leur « libération » pour les étudiants afro-américains.

Directement inspirée des écrits de Malcolm $\mathrm{X}^{17}$ et de Carmichael et Hamilton, cette revendication n'était cependant pas l'apanage des nationalistes noirs. L'idée qu'il était important pour la société américaine de reconnaître la richesse et la diversité des contributions apportées à l'histoire du pays par les communautés noires, mais également les groupes ethniques issus des différentes vagues d'immigration aux ÉtatsUnis, avait largement fait son chemin depuis l'apparition du mouvement pour les droits civiques sur la scène nationale. Témoin, par exemple, la modification adoptée le 26 juin 1967 de la section du code scolaire de l'État de l'Illinois portant sur l'enseignement de l'histoire américaine : l'amendement stipulait que ce dernier devait désormais « inclure une étude du rôle et des apports des noirs américains et d'autres groupes ethniques [...] à l'histoire de ce pays et de cet État ${ }^{18}$ "Un tel amendement avait été adopté par les législateurs de l'Illinois à des fins de reconnaissance du multiculturalisme de la nation 
américaine et de leur État, et non en vue d'une «libération » des élèves et étudiants afro-américains. Cette similarité des solutions prônées ne doit donc pas cacher la profonde différence entre les deux approches, mais elle explique la relative facilité avec laquelle certains administrateurs ont accueilli les demandes des étudiants concernant la création de départements d'études noires.

\section{Réactions des acteurs de l'institution}

La revendication de création d'un département d'études noires s'accompagnait de la dénonciation du racisme institutionnel des universités par les militants nationalistes noirs. Version universitaire de critiques similaires contre les autres institutions du pays par les tenants du black power, ces accusations n'en prirent pas moins de court bien des établissements. En effet, au-delà de leur rhétorique passionnée, les ultimatums transmis à l'administration des universités contenaient un message difficile à nier. La faible importance numérique des étudiants et des enseignants noirs sur les campus américains ne pouvait échapper à personne, même si la plupart des universités ne conservait pas de statistiques sur la composition raciale de leur corps étudiant et professoral à cette époque. Statistiques ou pas statistiques, il n'en restait pas moins que, de fait, les effectifs (étudiants et enseignants) des universités étaient loin d'être représentatifs de la composition démographique de la population et qu'aucun programme ne prenait en compte la contribution des afro-américains à la nation américaine ou la spécificité de leur expérience. Dans ces conditions, les actions de protestation des étudiants afro-américains les contraignirent à mener une véritable introspection, malgré leur refus d'admettre tout racisme de leur part. L'ampleur des changements sollicités par les étudiants activistes aurait mérité que les universités consacrent du temps et de la réflexion pour arriver à une solution satisfaisante pour l'ensemble de la communauté universitaire. Mais la manière dont ces revendications furent formulées ne leur en donna pas l'opportunité.

Introspection ne signifie pas pour autant levée des réticences. Si l'idée de création de nouveaux départements d'études noires pouvait être admise comme partie intégrante des mesures de correction (initiées par le mouvement pour les droits civiques) des injustices passées vécues par la communauté afro-américaine, leur présentation par des militants noirs n'hésitant pas à recourir aux ultimatums et aux menaces de violence, comme l'une des étapes d'une transformation radicale de l'institution universitaire pour la mettre au service de la communauté noire environnante suscita une montée des tensions, allant parfois jusqu'à la crise institutionnelle. Le climat sur les campus était d'autant plus tendu qu'à partir de 1968, le mythe de l'université " tour d'ivoire " avait vécu. Les administrateurs des établissements concernés ne pouvaient ignorer la radicalisation du mouvement noir dans les quartiers défavorisés et les émeutes raciales qui venaient d'enflammer les ghettos des grandes villes après l'assassinat de Martin Luther King début avril 1968. Aussi prirent-ils très au sérieux le spectre de la violence physique que firent planer sur les campus les ultimatums des étudiants afroaméricains. De fait, l'influence manifeste de l'idéologie du Black Power dans leurs écrits et, en particulier, l'utilisation d'expressions, telles que «by any means necessary» (par tous les moyens nécessaires), rappelant les appels à l'autodéfense armée de Malcolm X, semblaient indiquer que les activistes étaient prêts à tout pour obtenir satisfaction, $\mathrm{y}$ compris à faire usage de la violence. Aucun autre groupe étudiant militant n'avait encore usé de ce genre de menaces, jugées d'autant plus crédibles par les 
administrateurs que les étudiants activistes entretenaient des liens étroits avec leurs communautés.

Malgré les spécificités propres à chaque campus, la similarité des revendications des étudiants et l'enseignement tiré des autres conflits opposant étudiants et autorités universitaires expliquent une certaine similarité dans les réponses des universités du pays. À ce titre, le cas de San Francisco State College, mentionné en ouverture de cet article, est atypique du fait de la durée de la confrontation qui opposa les jeunes militants et l'administration. Dans la très grande majorité des cas, l'importance de la couverture médiatique consacrée aux manifestations d'activisme des étudiants noirs à partir de l'automne 1967 permit aux universités de se tenir au courant de ce qui se passait dans d'autres établissements et de tirer de ces expériences des leçons utiles pour leur propre situation, les orientant vers des solutions de compromis, généralement rapides, qui désamorcèrent la situation et donnèrent naissance aux départements d'études noires.

19 Ainsi, l'occupation de Howard University, la célèbre université noire de Washington, fin mars 1968 par un millier d'étudiants (sur 8200 au total) ${ }^{19}$ exigeant une réforme profonde du fonctionnement de l'établissement, y compris une refonte des programmes en vue de l'introduction d'enseignements «mieux en accord avec la pensée des étudiants noirs actuels » et le renforcement du rôle des étudiants dans le processus décisionnel de l'université, fut amplement suivie par la presse nationale ${ }^{20}$. Cette couverture médiatique fournit aux administrateurs des autres universités de nombreux détails quant à la solution choisie par les autorités de Howard : après avoir refusé de faire appel à la police pour évacuer les locaux occupés et menacé les étudiants de mesures disciplinaires, le board of regents de l'université décida de nommer un comité de réflexion sur les questions soulevées par les étudiants et dans un geste d'apaisement, de renoncer aux sanctions prévues par le règlement intérieur. Ainsi, sans accéder aux revendications et céder devant la pression de l'occupation, les autorités universitaires parvinrent néanmoins à briser la logique de confrontation établie par les manifestants. Les étudiants pouvaient également se dire satisfaits du résultat puisque, pour la première fois, l'administration de leur établissement reconnaissait suffisamment le bien-fondé de leurs revendications pour créer une commission chargée de les étudier plus avant ${ }^{21}$. Un mois plus tard, les événements de l'université Columbia à New York fournirent aux universités du pays l'exemple d'une réaction bien différente ${ }^{22}$. Elles purent constater notamment que l'intransigeance et le recours aux forces de police, loin de ramener le calme sur le campus, contribuait à rallier de nombreux membres de la communauté universitaire à la cause des étudiants radicaux.

Fortes de ces enseignements, les universités en grande majorité blanches du nord du pays (qui furent le site du plus grand nombre de revendications de créations de programmes d'études noires) adoptèrent, en règle générale, une attitude plus proche de celle des régents de Howard University que de celles de Grayson Kirk, le président de Columbia. Elles optèrent quasiment toutes pour la nomination d'une commission chargée d'étudier le bien-fondé éventuel des demandes qui leur étaient soumises. Ce faisant, les autorités universitaires à la fois accordèrent une légitimité aux étudiants afro-américains protestataires, pourtant très minoritaires sur le campus ou regroupés dans des organisations inconnues jusqu'alors de l'administration, et parvinrent à ramener le conflit sur leur propre terrain, dans le cadre d'une forme acceptée (la commission) de traitement des problèmes au sein de la communauté universitaire. Sans 
présager des conclusions de leurs commissions respectives, en offrant ainsi une première satisfaction aux deux parties en présence, les autorités universitaires firent le premier pas vers la sortie du conflit. Quelque temps plus tard, les universités touchées par ces mouvements de protestation se prononcèrent généralement pour la mise en place de mesures permettant d'accroître la proportion d'étudiants noirs sur leurs campus, pour la création de nouveaux programmes d'études noires et pour le recrutement accéléré d'enseignants et d'administrateurs afro-américains pour en assurer le fonctionnement.

Ce choix de la concession produisit généralement les effets souhaités de retour du calme. Dans certains cas, toutefois, comme à l'Université de Californie à Los Angeles (UCLA), la décision de l'administration d'accéder aux demandes des militants étudiants noirs ne parvint pas à protéger l'université contre l'irruption de la violence sur le campus : en janvier 1969, alors que l'université venait de créer le Centre d'études afroaméricaines (Center for Afro-American Studies), deux jeunes Black Panthers, Alprentice «Bunchy » Carter et John Huggins, dirigeants de la section de Los Angeles $\mathrm{du}$ BPP, furent assassinés par des membres du groupe nationaliste noir rival, United Slaves Organization (US) dirigé par Ron Karenga, dans une lutte pour décider de qui dirigerait le centre. Dans d'autres cas, dont le plus connu est celui de l'université Cornell, il fallut que la menace de la violence apparût clairement aux yeux de tous pour que la situation évolue et qu'une nouvelle structure consacrée aux études afroaméricaines voit le jour. Ainsi, en avril 1969, les militants de l'Afro-American Society de cet établissement basé à Ithaka dans l'État de New York, firent la une de la presse nationale et internationale en sortant du bâtiment qu'ils occupaient depuis huit jours armés de fusils mitrailleurs : cette action radicale (mais légale) poussa la communauté universitaire de Cornell à prendre enfin, après deux années de discussions vaines, en compte le sérieux des revendications des étudiants noirs et à donner son accord à la création de l'Africana Studies and Research Center placé sous la direction de James Turner $^{23}$.

22 Ces deux exemples de UCLA et Cornell mis à part, sur la plupart des campus, les tensions furent généralement moindres et les concessions accordées mirent fin au conflit ou tout au moins évitèrent son escalade. L'argument de la théorie des dominos avancée par certains administrateurs et enseignants pour refuser tout accord avec les étudiants radicaux ne sembla donc pas se vérifier. Le retour rapide du calme atteste la relative modération des étudiants noirs (en comparaison avec leurs pairs blancs): ayant obtenu satisfaction sur un grand nombre, voire la totalité, de leurs demandes, ils renoncèrent à aller plus loin dans la rébellion contre les autorités administratives, mirent fin au conflit et se consacrèrent désormais aux nouveaux défis créés par la création de ces nouveaux programmes dans une institution restée globalement inchangée.

\section{Premiers défis de l'institutionnalisation}

23 À peine la bataille de la création des départements d'études noires remportée que les premiers défis se posèrent. Trois questions devaient immédiatement être tranchées : le recrutement d'enseignants adéquats, l'orientation à donner au nouveau département et les stratégies permettant son institutionnalisation. 

départements dépassait de loin le nombre d'enseignants disponibles pour y enseigner. Cette pénurie relative s'avéra un problème essentiel pour l'orientation de ce nouveau champ disciplinaire. Dans la plupart des disciplines académiques, le diplôme le plus élevé est le doctorat. Dans les années soixante, des centaines d'afro-américains en étaient titulaires mais la grande majorité d'entre eux étaient déjà en poste dans les institutions noires du sud du pays. Pour enseigner dans les nouveaux départements du Nord, la seule autre source de docteurs noirs était alors des personnes originaires du continent africain ayant fait leurs études aux États-Unis, en Afrique, ou en GrandeBretagne. Avec la déségrégation de l'enseignement supérieur, les afro-américains firent leur entrée dans les institutions en grande majorité blanche dans la deuxième moitié des années soixante, mais ils n'atteignirent pas le niveau voulu de qualification avant plusieurs années. Désireuses d'embaucher immédiatement des professeurs afroaméricains, de nombreuses universités recrutèrent donc à leur place des professeurs africains, parce qu'ils étaient disponibles et remplissaient les critères de recrutement classiques des universités. Témoin d'une véritable évolution des mentalités, cette décision provoqua toutefois de grandes difficultés d'adaptation, tant de la part des étudiants que de la part de ces nouveaux enseignants, lesquels durent souvent combler très vite leurs lacunes en matière des spécificités de l'expérience afro-américaine. Certains s'adaptèrent remarquablement bien, comme Boniface Obichere, professeur nigérian qui enseigna à UCLA à la fin des années soixante. Dans certains cas, les universités, prises de désespoir dans leurs quêtes vaines pour recruter des professeurs qualifiés, décidèrent de se tourner vers des afro-américains sans diplôme ou tout au moins non-titulaires du doctorat, mais possédant un autre type de qualification : des militants locaux particulièrement compétents - tels Bayard Rustin, Eldridge Cleaver, Amiri Baraka et de nombreux autres - furent recrutés pour enseigner à l'université, souvent sous la forme de séries de conférences, mais parfois de manière plus permanente ${ }^{24}$. Bien entendu, le choix effectué par l'institution en matière de recrutement était décisif pour la définition de l'orientation du programme ou du département - soit tourné vers les études africana et diasporiques, soit tourné vers la communauté.

Nés d'un mouvement populaire soucieux de répondre aux besoins de la communauté noire, à l'université mais aussi et surtout au-delà, dans les quartiers défavorisés, les départements d'études noires tentèrent, dès leur création, de combiner leur ambition scientifique et académique et leur objectif de service à la communauté afro-américaine. Sur certains campus, les étudiants et professeurs à l'origine de cette demande de création de nouvelles structures tentèrent même de forcer l'institution universitaire à mettre l'accent sur ce second objectif, placé au cœur de leur projet. Tel fut le cas de l'Université de l'Illinois à Chicago où un projet de création d'un Centre d'études ethniques proposant un master en études urbaines, en lien direct avec les besoins de la communauté locale, fut sérieusement discuté pendant plusieurs mois, avant d'être abandonné sous le double effet d'une forte résistance de l'administration et d'importantes divisions des défenseurs des différents groupes minoritaires en présence à Chicago en 1969. En lieu et place du centre pionnier envisagé initialement, un département d'études noires tourné, de manière traditionnelle donc acceptable pour l'institution universitaire, vers la recherche et l'enseignement vit finalement le jour en $1971^{25}$. Cet échec illustre bien la résistance de l'institution universitaire à accepter une remise en cause fondamentale de sa fonction sociale et est typique de l'orientation 
académique qui finit par l'emporter dans les départements d'études noires des universités américaines, malgré une volonté toujours présente de leurs acteurs de s'efforcer de conserver une pertinence et une utilité pour la population afroaméricaine en dehors de la communauté universitaire.

Une fois ces premiers défis de la naissance et de l'orientation initiale relevés, différentes stratégies, typiques de la première vague de création des départements d'études noires, furent suggérées, dans la première moitié des années 1970, par les acteurs de ces nouvelles structures pour institutionnaliser les études noires tout en essayant de maintenir des liens, mêmes moindres que ceux initialement espérés, avec la communauté noire environnante, dans un contexte politique marqué par un retour de plus en plus net du conservatisme depuis l'arrivée de Nixon à la Maison blanche début 1969. Certes les démocrates avaient réussi à maintenir leur majorité (en la renforçant même à la Chambre des Représentants mais en perdant quelques sièges au Sénat) au Congrès aux élections de mi-mandat de 1970, mais ils avaient renoncé à faire campagne sur les questions sociales (et $a$ fortiori raciales) pour se concentrer sur les difficultés économiques que traversait le pays et qu'ils imputaient à la mauvaise gestion de l'administration Nixon. En ce début des années soixante-dix, les programmes de promotion de la justice sociale et raciale typiques de l'Amérique de la Grande Société de Johnson étaient attaqués de toutes parts par une « majorité silencieuse » conservatrice de plus en plus déterminée à faire valoir ses propres intérêts et ses droits, en particulier, face à la minorité afro-américaine, tandis que les organisations nationalistes noires comme le Black Panther Party étaient réduites à néant ou à l'impuissance sous les coups d'une campagne massive de répression politique ${ }^{26}$. L'idéal de colorblindness commença alors à revenir sur le devant de la scène, revisité par le conservatisme ambiant, pour justifier les efforts conjoints d'hommes politiques et d'acteurs de la communauté universitaire pour limiter au maximum la portée de la révolution que constituait l'introduction des études noires à l'université américaine.

Dans ce contexte, les premiers enseignants de ces programmes défendirent et mirent en pratique toute une série de stratégies qu'ils adaptèrent aux réalités et aux rapports de force en vigueur dans leurs universités pour assurer leur pérennité. Il s'agissait avant tout ${ }^{27}$ d'exiger le statut de "département " pour les études noires, une étape essentielle pour garantir le contrôle des procédures et des décisions de recrutement, de titularisation, de contrôle du budget de fonctionnement et du programme enseigné. En cas d'impossibilité due aux conditions politiques ou à la taille de l'institution, le statut de «programme » était revendiqué pour contrôler, au moins, les cours enseignés. Une deuxième revendication consistait à n'accepter qu'avec une grande prudence les affectations d'enseignants conjointes sur deux départements, par exemple études noires et histoire, afin de garantir que les départements d'études noires soient traités sur un pied d'égalité avec les autres structures académiques ${ }^{28}$. Pour maintenir la pertinence des programmes pour la communauté noire alentour, les enseignants développèrent souvent un système de stages dans des organisations et associations agissant au niveau local, en lien avec les cours enseignés dans les sciences humaines, les arts, les sciences sociales et naturelles. Le but de ces stages était aussi de présenter les étudiants et leurs enseignants aux habitants noirs des quartiers environnants pour que la communauté soit au courant de ce qui se passe sur le campus et de fournir aux étudiants l'opportunité d'évaluer les possibilités de travail pendant leurs années de scolarité undergraduate ou postgraduate. Enfin, sur chaque campus touché par le mouvement des études noires, le centre culturel afro-américain jouait un rôle essentiel 
dans l'institutionnalisation des études noires en maintenant un lien entre les activités extracurriculaires et la recherche, la pédagogie et l'enseignement grâce à l'organisation de séries de films et autres événements culturels et sociaux. Ces centres avaient joué un rôle historique très fort dans la création des départements d'études noires en offrant un havre de paix aux étudiants noirs souvent aux prises avec du ressentiment et de l'hostilité sur leurs campus majoritairement blancs. Avec le retour d'une atmosphère moins tendue sur les campus au début des années 1970, les centres culturels continuèrent de permettre de lier études et autres aspects de la vie étudiante, d'une manière que l'on rencontre rarement ailleurs sur les campus américains, même aujourd'hui. Deux autres revendications consistaient, d'une part, à développer la recherche, l'enseignement et la pratique de la musique, de l'art, du théâtre et de la danse africains et afro-américains, des champs négligés par les institutions universitaires et, d'autre part, à développer les offres de cours sur l'histoire, la littérature, la politique et l'économie de l'Afrique et de la diaspora. Enfin, le suivi des diplômés de ces premiers programmes jusqu'à leur insertion dans la vie active et le développement d'une base d'alumni, ces anciens étudiants qui continuent de soutenir, notamment financièrement, leur alma mater et leur programme de formation, constituaient deux stratégies complémentaires permettant de démontrer l'utilité du choix d'un tel programme et d'obtenir un soutien de l'université pour les étudiants du programme et le département lui-même.

Ces différentes stratégies permirent, sur la plupart des campus, d'ancrer ces nouveaux programmes créés dans la durée en en faisant des unités dynamiques attirant un nombre croissant d'étudiants. Progressivement, au fur et à mesure que le nombre d'étudiants noirs détenteurs du doctorat augmentait, il devint plus facile pour les universités de trouver des enseignants répondant aux normes les plus sélectives de recrutement pratiquées. Cette reconnaissance institutionnelle, basée sur la triple qualité des enseignements dispensés, de la recherche produite et du corps professoral, était essentielle pour la pérennité des départements, et pas uniquement pour attirer des étudiants d'un bon niveau en nombre suffisant. Depuis les premiers jours (et jusqu'à aujourd'hui), l'un des problèmes fondamentaux pour le maintien et le développement des départements d'études noires était en effet l'obtention d'un soutien financier suffisant, permettant de continuer de proposer une offre de cours de qualité et d'attirer des enseignants réputés grâce à une politique salariale compétitive. La dépendance financière des départements d'études noires envers l'administration de leur université était si cruciale qu'elle força les nouvelles structures à évoluer vers des positions de moins en moins radicales, plus propres à garantir l'allocation de fonds au sein d'une institution où, dans le contexte de la crise économique des années 1970, les ressources des universités - nombre d'étudiants payant des droits, financements de l'État fédéral et des États, financements extérieurs, notamment des anciens étudiants s'amenuisaient. La manne financière providentielle que constitua pour les programmes d'études noires le financement apporté jusqu'au milieu des années 1970 par la Fondation Ford participa de cette même évolution ${ }^{29}$ : prolongement de son action menée en faveur des droits civiques depuis les années 1950, cette décision de l'organisme philanthropique de soutenir financièrement le développement des programmes d'études noires à l'université finit de les faire évoluer loin du radicalisme des toutes premières années: en choisissant de concentrer son aide sur les départements de quelques établissements d'élite, tel celui de l'université Harvard, la Fondation Ford contribua à accélérer le processus de sélection naturelle entre 
structures concurrentes : alors que le nombre d'étudiants noirs n'était pas expansible à l'infini et que le nombre de chercheurs réputés était lui aussi limité, les différents départements se mirent à rivaliser les uns avec les autres pour les attirer, une course qui favorisa nécessairement les structures les mieux dotées. En outre, la Fondation Ford choisit de privilégier les programmes à orientation académique classique, au détriment des programmes à orientation communautaire et/ou nationaliste, poussant ainsi l'ensemble des structures désireuses de bénéficier de son aide vers des positions de plus en plus éloignées du black power. Or au début des années 1970, ces deux types de programmes d'études noires étaient déjà sur la défensive sous l'effet conjugué du retour en force d'une idéologie conservatrice colorblind privilégiant la valeur du succès individuel sur les progrès du groupe et du reflux généralisé du mouvement du black power du fait des limites de son action dans les quartiers défavorisés et de la campagne massive de répression qui s'abattit sur lui. Dans ces conditions, alors que les universités avaient été le dernier bastion de l'idéologie du black power, les défis posés par l'institutionnalisation des départements d'études noires - et en particulier le problème sans cesse renouvelé de leur financement - sonnèrent le glas de toute orientation radicale, directement héritière du mouvement social des années soixante qui leur avait donné naissance.

\section{Conclusion}

Ces premiers défis liés à la nécessaire adaptation d'un mouvement social radical aux logiques inhérentes à l'institution universitaire continuèrent de marquer les départements d'études noires pendant de nombreuses années après la phase de dynamisme initial de la fin des années 1960 et du début des années 1970. Pendant près de 15 ans après leur apparition dans le paysage de l'enseignement supérieur américain, ces structures souffrirent d'une crise de légitimité prolongée les obligeant à dépenser leurs forces pour se défendre contre ceux, au sein de l'université comme ailleurs, qui souhaitaient les voir disparaître, soit qu'elles étaient accusées d'offrir un enseignement au rabais sur des sujets de moindre importance, soit qu'on leur opposait l'argument liberal contre l'existence même de structures séparées. C'est notamment pour répondre à ces accusations que le National Council for Black Studies, fondé en 1975 par des professeurs d'études afro-américaines, définit en 1981 un programme commun (core curriculum) de contenus de qualité à enseigner dans chaque département et constituant la base d'une accréditation accordée par l'organisation professionnelle ${ }^{30}$. Source d'une cohérence accrue et de normes plus élevées dans les programmes existants, cette initiative du NCBS permit de faire taire une partie des critiques à une époque où la révolution reaganienne à l'œuvre entendait battre activement en brèche tout ce qui pouvait être perçu comme un héritage du libéralisme des années 1960. Si l'avènement du politiquement correct à l'université parut un temps pouvoir offrir aux départements d'études noires une trêve bienvenue dans les attaques frontales (voire racistes) qu'ils subissaient depuis les années 1970, les "guerres culturelles" qui l'accompagnèrent marqua une recrudescence des critiques dans le contexte d'une Amérique très largement gagnée à l'idéologie conservatrice ambiante ${ }^{31}$. Si certains programmes connurent un regain d'intérêt et de renommée à cette époque, notamment lorsque l'université Harvard confia à Henry Louis Gates Jr. le soin de former une "dream team » pour relancer son centre d'études afro-américaines en recrutant les meilleurs spécialistes, dans l'ensemble du pays, l'évolution depuis les 
années 1990 est à la diminution du nombre de programmes autonomes avec réintégration des études noires dans des départements académiques classiques (tels les départements d'histoire, de science politique ou de sociologie). Dans ce contexte, la crise financière et économique actuelle qui touche depuis ces dernières années les universités américaines et les force à réduire considérablement leurs activités ne saurait manquer de poser de nouveaux défis considérables à des structures qui restent aujourd'hui encore largement contestées au sein de l'institution universitaire.

\section{BIBLIOGRAPHIE}

ALDRIDGE Delores P. and Carlene YOUNG (eds.), Out of the Revolution: The Development of Africana Studies, Lanham (MD), Lexington Books, 2003.

ASANTE Molefi et Ama MAZAMA (eds.), The Encyclopedia of Black Studies, Thousand Oaks (CA), Sage Publications, 2005.

D'SOUZA Dinesh, Illiberal Education: The Politics of Race and Sex on Campus, Glencoe (IL), Free Press, 1991.

GLASKER Wayne, Black Students in the Ivory Tower : African American Student Activism at the University of Pennsylvania, 1967-1990, Amherst et Boston, University of Massachusetts Press, 2002.

JOSEPH Peniel E., "Dashikis and Democracy : Black Studies, Student Activism, and the Black Power Movement", The Journal of African American History, vol. 88, No. 2, The History of Black Student Activism (Spring, 2003), p. 182-203.

NORMENT Nathaniel, Jr. (ed.), The African American Studies Reader, Durham (N.C.), Carolina Academic Press, 2001.

OGBAR Jeffrey. O. G., Black Power : Radical Politics and African American Identity, Baltimore, Johns Hopkins University Press, 2004.

ROJAS Fabio, From Black Power to Black Studies : How a Radical Social Movement Became an Academic Discipline, Baltimore, Johns Hopkins University Press, 2007.

SYKE Charles, Profscam : Professors and the Demise of Higher Education, Washington, D.C., Regnery Publishing, 1989.

VAN DEBURG William, New Day in Babylon: The Black Power Movement and American Culture, 1965-1975, Chicago, University of Chicago Press, 1992.

WILLIAMSON Joy Ann, Black Power on Campus. The University of Illinois, 1965-1975, Urbana et Chicago, University of Illinois Press, 2003.

\section{NOTES}

1. Plusieurs études ont été consacrées à cet événement majeur dans l'histoire de l'enseignement supérieur américain: voir notamment l'ouvrage classique de William Orrick, Shut It Down! A College in Crisis, Aurora (IL), Aurora Press, 1970 et plus récemment le chapitre 3 « Revolution at 
San Francisco State College » du livre de Fabio Rojas, From Black Studies to Black Power: How a Radical Social Movement Became an Academic Discipline, Baltimore, Johns Hopkins University Press, 2007, p. 45-92.

2. Les estimations les plus conservatrices, tirées des résultats d'un sondage effectué dans le cadre de l'étude de Philip T.K. Daniel et Asmasu Zike, « Black Studies Four-Year College and University Survey ", Center for Minorities Studies, Northern Illinois University, mai 1983, sont comprises entre 130 et 160 . Les estimations les plus hautes parlent de 500 programmes créés : Cf. Martin Weston, «Black Studies: Dead or Alive?»Essence, 5 (août 1974), p. 57 cité dans Alan Colón, «Black Studies and Historically Black Colleges and Universities", in Delores P. Aldridge et Charlene Young (dir.), Out of the Revolution: The Development of Africana Studies, Lanham, Lexington Books, 2000, p. 287-313.

3. Malcolm X, «The Ballot or the Bullet », discours prononcé le 3 avril 1964 et repris dans Alexander Bloom et Wini Breines (dir.), op. cit., p. 105-108.

4. Wayne Glasker, Black Students in the Ivory Tower: African American Student Activism at the University of Pennsylvania, 1967-1990, Amherst et Boston, University of Massachusetts Press, 2002, p. 11-12.

5. Fabio Rojas, From Black Power to Black Studies, op. cit., p 22-44.

6. Voir l'article de Lawrence P. Crouchett, "Early Black Studies Movements " in Nathaniel Norment (ed.), The African American Studies Reader, p. 192-198 ; voir également Peniel E. Joseph, "Dashikis and Democracy: Black Studies, Student Activism, and the Black Power Movement", The Journal of African American History, vol. 88, n², numéro special sur l'activisme des étudiants noirs, printemps 2003, p. 182-203.

7. Carter G. Woodson, The Mis-Education of the Negro, Trenton (NJ), Africa World Press, 1933.

8. Cf. William E. Nelson, « Black Studies, Student Activism and the Academy » in Delores Aldridge et Carlene Young (dir.), op. cit., p. 19-91.

9. Une version plus détaillée de ce développement figure dans Caroline Rolland-Diamond, Chicago : le moment 68. Territoires de la contestation étudiante et répression politique, Paris, Syllepse, 2011, chapitre 4.

10. Le chiffre exact est de $22,9 \%$ pour une population totale de plus de 3,5 millions en 1960 . (Statistical Abstract of the United States, 1966 (http://www2.census.gov/prod2/statcomp/ documents/1966-02.pdf). En 1970, cette proportion s'était élevée à 32,7 \% (pop. tot. : 3367000 habitants). (Statistical Abstract of the United States, 1971 (http://www2.census.gov/prod2/ statcomp/documents/1971-02.pdf).

11. Chicago Maroon [journal étudiant de l'Université de Chicago], 29 septembre 1967.

12. Doug McAdam, Political Process and the Development of Black Insurgency, Chicago, University of Chicago Press, 1983, p. 36-59. Voir aussi Sidney Tarrow, Power in Movement: Social Movements and Contentious Politics, Cambridge, Cambridge University Press, 1998.

13. Cf. Marvin W. Peterson et al., Black Students on White Campuses: The Impacts of Increased Black Enrollments, Ann Arbor (MI.), Institute for Social Research, 1978, p. 23-42.

14. Durward Long, "Black Protest ", in Julian Foster et Durward Long (dir.), Protest ! Student Activism in America, New York (N.Y.), Morrow, 1970, p. 459-482.

15. Voir surtout President's Commission on Campus Unrest, Government Printing Office, 1970; W. Glasker, op. cit. ; James McEvoy et Abraham Miller (dir.), Black Power and Student Rebellion, Belmont (CA.), Wadsworth, 1969, ou plus récemment Richard P. McCormick, The Black Student Protest at Rutgers, New Brunswick (N.J.), Rutgers University Press, 1990.

16. Sur les cas de Brown, voir Rhett Jones "Dreams, Nightmares, and Realities: Afro-American Studies at Brown, 1969-1986" p. 33-50 et de Harvard, voir Martin Kilson "From the Birth to a Mature Afro-American Studies at Harvard, 1969-2002", p. 59-75, tous deux extraits de Lewis R. Gordon et Jane Anna Gordon (dir.), A Companion to African-American Studies, Malden (MA), 
Blackwell Publishing, 2006. Pour une étude comparée de la situation à l'Université de l'Illinois à Chicago et à l'Université de Chicago, voir Fabio Rojas, op. cit., p. 93-129.

17. « Malcolm X on Afro-American History ", discours prononcé le 24 janvier 1965 à la réunion de l'Organisation de l'Unité Afro-Américaine, reproduit dans Betty Shabazz (dir.), Malcolm X on Afro-

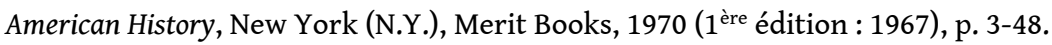

18. Illinois School Code, Section 27-21, amendé le 26 juin 1967, cité dans John P. Mercer, «Ethnic Group Education ", Illinois Journal of Education, 61 (4), avril 1970, p. 3.

19. Sur les attitudes des étudiants pendant ce mouvement de protestation, voir l'étude de Sophia F. McDowell, Gilbert A. Lowe, Jr. et Doris A. Dockett, «Howard University's Student Protest Movement ", The Public Opinion Quarterly, Vol 34, No. 3 (Autumn, 1970), p. 383-388.

20. A Chicago, par exemple, voir les articles du Chicago Tribune, «Force Negro University to Close Doors » (21/3/1968), « Sues to Force Opening Doors of Howard U. » (22/3/1968) et «Students End 5-Day Siege at Howard U.» (24/3/1968). Voir aussi l'article de Susan Jacoby, «Academic, Racial Issues Stir Howard Unrest », The Washington Post, Times Herald, 24 mars 1968.

21. Cf. Lawrence B. de Graaf, « Howard: The Evolution of Black Student Revolt », in Julian Foster et Durward Long (dir.), op. cit., p. 319-344.

22. Sur les événements de ce campus, voir le rapport de la Commission officielle d'enquête dirigée par Archibald Cox, «The Cox Commission Report. Crisis at Columbia. Report of the FactFinding Commission Appointed to Investigate the Disturbances at Columbia University in April and May 1968 ", Vintage Books, New York, Random House, 1968; "Six Weeks that Shook Morningside ", Columbia College Today, printemps 1968 et la biographie de l'un des leaders du mouvement, Mark Rudd, Underground: My Life with SDS and The Weathermen, New York, HarperCollins, 2009.

23. L'histoire de la création du Centre d'études afro-américaines de UCLA est présentée sur son site web: Ralph J. Bunche Center for African American Studies at UCLA, URL: http:// www.bunche.ucla.edu/newsite/index.html (consulté le 7 mars 2011) et celle de l'Africana Center de Cornell dans l'ouvrage Donald Alexander Downs, Cornell '69: Liberalism and the Crisis of the American University, Ithaca (NY), Cornell University Press, 1999.

24. Molefi Kete Asante, "Sustaining Africology: On the Creation and Development of a Discipline », in Gordon et Gordon, op. cit., p. 20-32, en particulier pages 22-23.

25. Cf. Fabio Rojas, op. cit., p. 104-108 et p. 112-116.

26. Sur la répression politique des organisations nationalistes noires, voir les rapports des enquêtes officielles publiées respectivement sous les références : U.S. Congress, House of Representatives, Committee on Internal Security, Civil Rights and Constitutional Rights Subcommittee, Hearing on FBI Counterintelligence Programs, 93rd Congress, 2nd session, Washington, U.S. Government Printing Office, 1974 et U.S. Congress, House of Representatives, Committee on Internal Security, Hearings on Domestic Intelligence Operations for Internal Security Purposes, 93rd Congress, 2nd session, Washington, U.S. Government Printing Office, 1974. Voir aussi les deux ouvrages de Ward Churchill et Jim Vander Wall, Agents of Repression: The FBI's Secret Wars against the Black Panther Party and the American Indian Movement, Boston, South End Press, 1988 et The COINTELPRO Papers: Documents From the FBI's Secret Wars Against Dissent in the United States, Boston, South End Press, 1990.

27. Ce développement s'inspire de Johnnella E. Butler, «Black Studies and Ethnic Studies », in Gordon et Gordon, op. cit., p. 76-95.

28. Ces mêmes revendications restent défendues par le National Council for Black Studies, organisme professionnel créé en 1975 pour soutenir l'institutionnalisation de ces programmes et leur développement. Voir le site Web de l'organisation : http://www.ncbsonline.org.

29. Cf. Fabio Rojas, op. cit., 130-166. 
30. Cf. Site web du NCBS, http://www.ncbsonline.org. Le core curriculum a été renouvelé en 1991. 31. Deux exemples d'ouvrages typiques des attaques de la période des « guerres culturelles » sont Charles Syke, Profscam: Professors and the Demise of Higher Education, Washington, D.C., Regnery Publishing, 1989 et Dinesh D'Souza, Illiberal Education: The Politics of Race and Sex on Campus, Glencoe (IL), Free Press, 1991.

\section{RÉSUMÉS}

Cet article examine les conditions historiques d'émergence des départements d'études noires dans les universités américaines à partir de l'année 1968 et les premiers défis que ces nouvelles structures rencontrèrent dans leur processus d'institutionnalisation. Plus précisément, il revient sur le contexte historique de l'arrivée de l'idéologie du black power sur les campus américains à la fin des années 1960 (conjonction des trois facteurs décisifs de la désillusion causée par les limites du mouvement pour les droits civiques, de la popularité grandissante du black power chez les jeunes afro-américains et de l'augmentation du nombre d'étudiants noirs sur des campus majoritairement blancs). Cet article examine ensuite la nécessaire adaptation de cette idéologie nationaliste noire, aspirant à améliorer les conditions de vie et à accroître le pouvoir de la communauté noire dans les quartiers défavorisés des grandes villes, à une institution universitaire restée largement inchangée après plusieurs années de protestation étudiante. S'appuyant sur plusieurs exemples d'universités ayant été touchées par le mouvement de création de départements d'études noires, cet article passe ensuite en revue les défis auxquels ont été confrontés les étudiants et enseignants noirs favorables à leur introduction et les différentes stratégies qu'ils ont mises en œuvre pour favoriser leur institutionnalisation et leur pérennité, dans le contexte de la montée du conservatisme politique sous la présidence Nixon. Il soutient la thèse selon laquelle les exigences propres à l'acceptation de ces nouveaux départements par l'institution universitaire (gage de financements suffisants pour assurer leur fonctionnement) avaient poussé ces derniers à renoncer à toute orientation communautaire ou nationaliste au profit d'une structure académique traditionnelle et que cette évolution devint irrémédiable du fait de l'évolution politique et sociale du pays vers des positions conservatrices mêlant idéal social de colorblindness (l'indifférence à la couleur de peau) et valorisation du progrès individuel.

This article examines the historical conditions that shaped the development of Black Studies departments within American universities in the late 1960s and 1970s, focusing on the initial challenges these new institutions faced. It begins by viewing these circumstances in relation to the rise of Black Power ideology on campuses throughout the United States in the late 1960s, highlighting the manner in which ideas of black nationalism aimed at improving ghetto conditions were adapted to university institutions that had remained until then largely unaffected by years of student protests. Based on several case studies, my analysis seeks to highlight the different strategies adopted by students and faculty members to promote the institutional legitimacy of Black Studies departments in a context of conservative backlash during the Nixon presidency. I argue that practical exigencies related to the drive for institutional acceptance and financial viability forced these actors to abandon their nationalist or community orientations and to move towards a more traditional academic outlook-a change 
that became irreversible within an increasingly conservative national political landscape dominated by ideals of colorblindness and individualism.

Este artículo examina las condiciones históricas que permitieron el desarrollo de los departamentos especializados en estudios sobre los afroamericanos en las universidades americanas durante los años 1960 y 1970. El análisis se centra en los desafíos y retos que inicialmente existieron en ese proceso de institucionalización. Concretamente, se analiza el contexto histórico de la llegada de la ideología del Black Power a los campus americanos a finales de 1960 (conjunción de tres factores decisivos; la desilusión causada por los límites del movimiento por los derechos civiles, la creciente popularidad del Black Power entre los jóvenes afro-americanos y finalmente, el creciente número de estudiantes negros en las universidades, hasta entonces predominantemente blancas). El artículo examina la forma en la que se adaptó la ideología nacionalista negra, (que tenía como fin, mejorar las condiciones de vida y darle más poder a la comunidad negra de los suburbios de las grandes ciudades), a la protesta estudiantil de una universidad. Sobre la base de varios ejemplos de universidades que siguieron el movimiento y aceptaron la creación de un departamento de Estudios Negros, este artículo analiza los desafíos a los que se enfrentaron los estudiantes y profesores negros que apoyaban este movimiento y las diferentes estrategias que pusieron en marcha para dinamizar la institucionalización y sostenibilidad de estos nuevos departamentos, en un contexto de política conservadora, promovida por la presidencia de Nixon.

Finalmente, se demuestra cómo los requisitos demandados por la Universidad para aceptar estos nuevos departamentos (entre ellos, garantizar una financiación suficiente para llevar a cabo sus proyectos) llevaron, a estos últimos, a pasar de una orientación "comunitaria- nacionalista,"a una estructura académica tradicional. Esta tendencia se hizo irreversible debido a la evolución política y social del país hacia posiciones más conservadoras que defendían un ideal social de "colorblindnes" (indiferencia hacia el color de la piel) y promovían el progreso individual.

\section{AUTEUR}

\section{CAROLINE ROLLAND-DIAMOND}

Maître de conférences en histoire américaine à l'Université Paris Ouest Nanterre. Ses axes de recherche portent sur l'histoire sociale, l'histoire politique et l'histoire urbaine des États-Unis au XXe siècle, ainsi que sur l'histoire des mouvements étudiants américains depuis les années 1960, l'histoire de l'institution universitaire, la théorie des mouvements sociaux et l'histoire de la répression politique. Elle est l'auteur de Chicago : le moment 68 . Territoires de la contestation étudiante et répression politique (Paris: Syllepse, 2011) et a co-dirigé (avec Robi Morder) Etudiant(e)s du monde en mouvement. Migrations. Cosmopolitisme et internationales étudiantes (Paris, Syllepse, 2012). 